\title{
The impulsive motion of a liquid resulting from a particle collision $\dagger$
}

\author{
By R. ZENIT AND M. L. HUNT \\ Division of Engineering and Applied Sciences, California Institute of Technology, \\ Pasadena, CA 91125, USA
}

(Received 2 February 1998 and in revised form 8 June 1998)

When two particles collide in a liquid, the impulsive acceleration due to the rebound produces a pressure pulse that is transmitted through the fluid. Detailed measurements were made of the pressure pulse and the motion of the particles by generating controlled collisions with an immersed dual pendulum. The experiments were performed for a range of impact velocities, angles of incidence, and distances between the wall and the pairs of particles. The radiated fluid pressure was measured using a high-frequency-response pressure transducer, and the motion of the particles was recorded using a high-speed digital camera. The magnitude of the impulse pressure was found to scale with the particle velocity, the particle diameter and the density of the fluid. Additionally, a model is proposed to predict the impulse field in the fluid based on the impulse pressure theory. The model agrees well with the experimental measurements.

\section{Introduction}

In particulate flows the collisional particle pressure, or granular pressure, results from the interactions between individual particles and solid boundaries. Many researchers have developed models for the particle pressure, but none had been corroborated experimentally due to the difficulties encountered in its measurement. In a recent investigation, Zenit, Hunt \& Brennen (1997) reported measurements of the collisional particle pressure for solid-liquid flows. The pressure measured was found to be composed of two contributions: direct collisions of particles against the surface (Zenit \& Hunt 1998), and a pressure contribution from particle-to-particle collisions that was transmitted through the fluid.

The present paper is an analysis of the second, or radiated, contribution to the particle pressure. When a collision occurs, the impulsive acceleration of the surface of the particle caused by the rebound produces a pressure increase in the liquid. This pressure front is transmitted through the fluid from the surface of the particle to the entire flow field. The intensity of the pressure pulse is related to the collision velocity, the size and shape of the particles, and the density of the fluid. According Zenit et al. (1997), this pressure contribution accounts for only $5 \%$ to the total particle pressure (in the case of glass spheres); however, particle collisions are a source of fluid pressure disturbances which may have an effect on the stability of a liquid-solid mixture.

The impulsive motion of immersed surfaces has been long studied (Lamb 1945). Its development has been motivated recently by problems related to water wave impacts against coastal structures (Cooker \& Peregrine 1995). The impulsive motion 


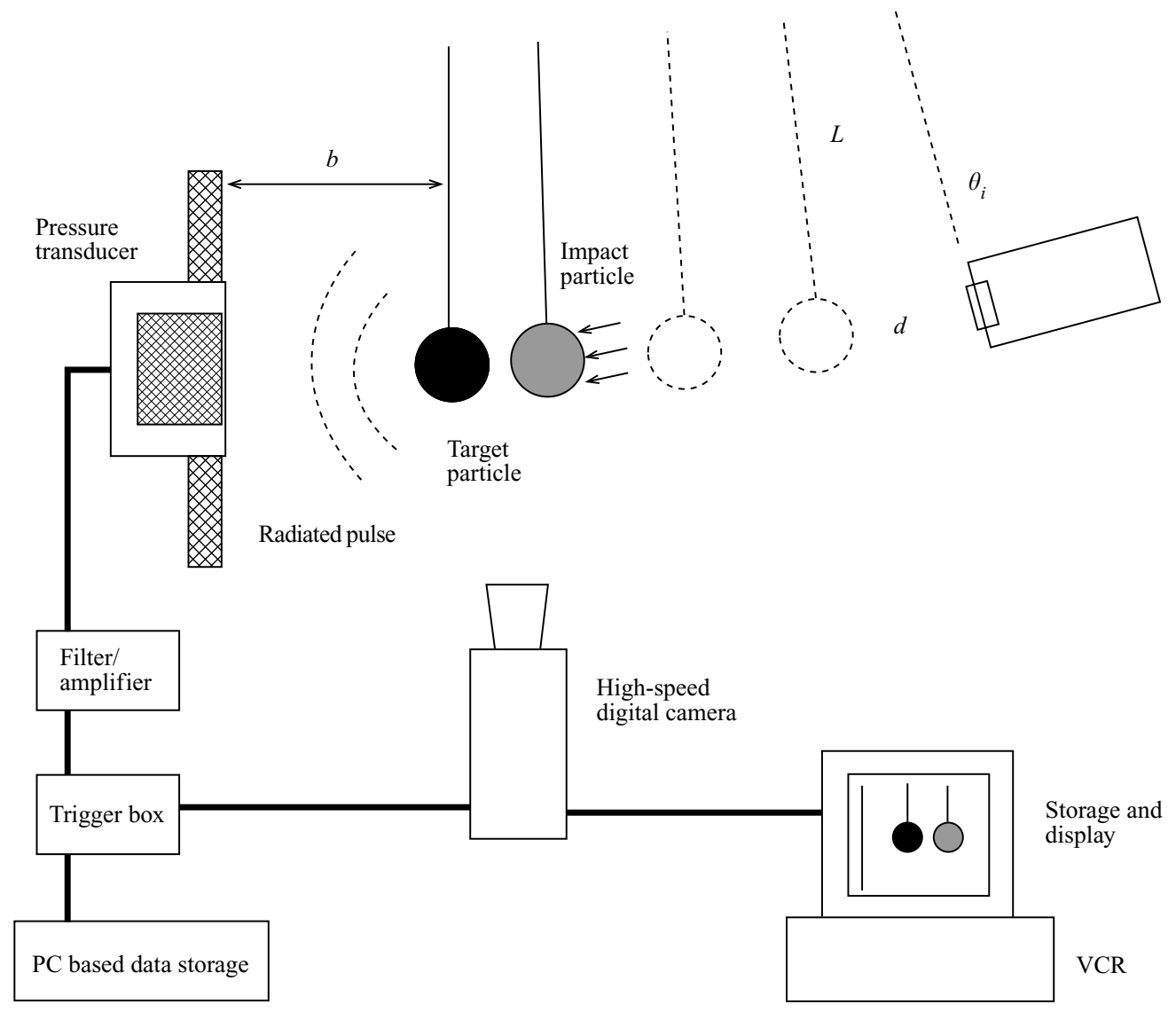

FIGURE 1. Experimental setup of the dual pendulum to measure the fluid pressure produced by a collision.

of a sphere has also been studied in the small Reynolds number range by Mei \& Lawrence (1996) and Lovalenti \& Brady (1995).

Experiments involving binary collisions of particles were conducted, and preliminary results can be found in Zenit \& Hunt (1997). To provide a range of fluid accelerations, particles of different densities were tested for varying impact velocities. The results are presented in terms of the impulse pressure. Additionally, to corroborate the experimental measurements, a model based on impulse pressure theory is proposed, indicating good agreement with the experiments.

\section{Experimental setup}

A binary collision experiment was used to obtain a quantitative measure of the pressure front generated by the collision of particles. The experimental arrangement is shown in figure 1. A dual pendulum was built to generate controlled collisions. A particle was suspended (the target particle) at a certain distance, $b$, from a wall. Flush mounted to the wall was a pressure transducer with a frequency response of $500 \mathrm{kHz}$ and a sensitive surface of $3.76 \mathrm{~mm}$ in diameter. A second particle (the impact particle) was released starting from rest at some initial angle. The impact particle accelerated towards the target particle resulting in a collision that produced a 


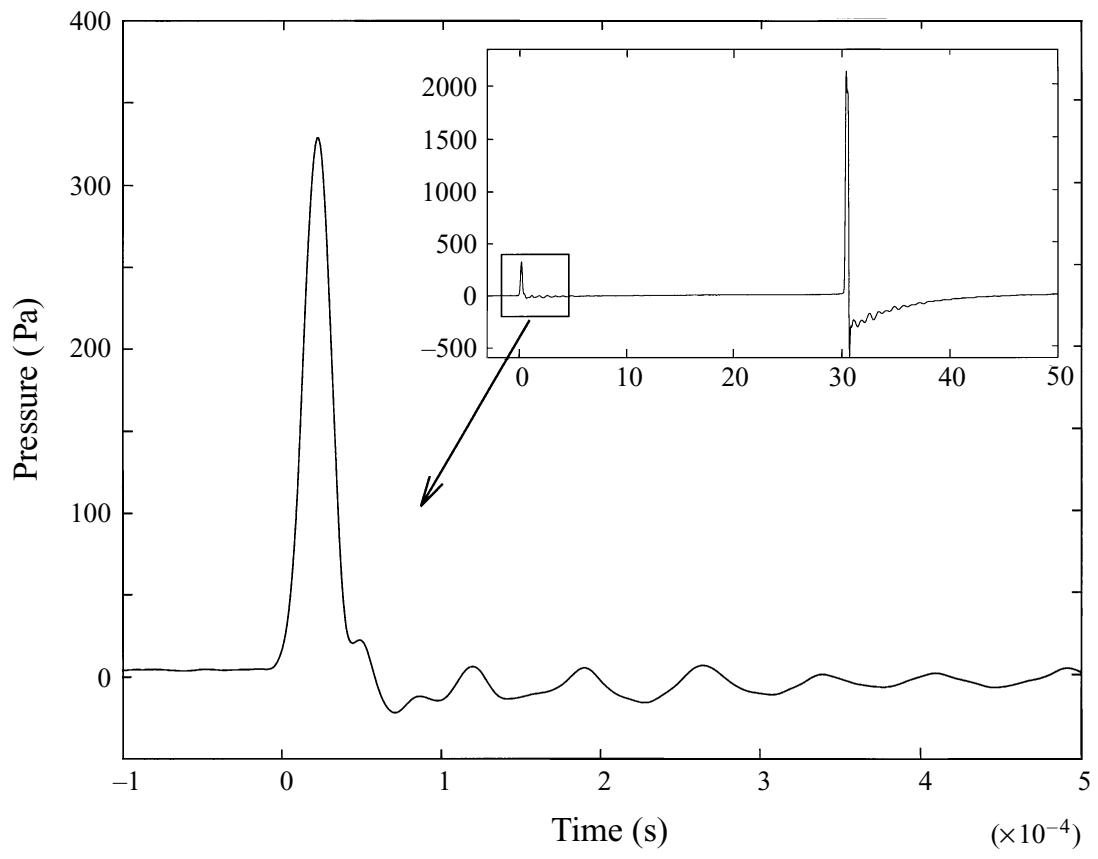

FIGURE 2. Typical pressure trace produced by the impulsive acceleration of the fluid after a collision of two $6 \mathrm{~mm}$ glass particles in water. The zero time corresponds to the time when the binary collision occurred (first spike). The second spike, shown in the insert, occurs at approximately $30 \times 10^{-4} \mathrm{~s}$ and is the result of the target particle impacting the pressure transducer.

sudden acceleration of the target particle and generated an impulsive pressure front. The pressure pulse was transmitted through the fluid from the surface of the target particle to the pressure transducer. The motion of both particles was recorded using a high-speed digital camera, which allowed the position and velocity to be measured moments after the impact occurred. The velocity of the impact particle, $u_{i m p}$, and the velocity acquired by the target particle, $u_{\tau}$, were calculated from the measured displacement of the particle centres before and after the collision, respectively. Since the maximum framing rate of the digital camera was 500 frames per second, the velocity is calculated at time increments of $2 \mathrm{~ms}$. The particle velocities obtained for a given initial angle of release were reproducible within $5 \%$ of variance. Most of the experiments were performed using pairs of identical particles.

A typical pressure pulse is shown in figure 2. For this particular case the distance from the centre of the target particle to the wall, $b$, was $3.6 \mathrm{~mm}$. The velocity of the impact particle, $u_{i m p}$, was approximately $120 \mathrm{~mm} / \mathrm{s}$. Since the data acquisition and the imaging systems were synchronized, it was possible to verify that the pressure recorded corresponded to the moment when the collision occurred and that neither of the particles were in contact with the transducer at the beginning of the pressure pulse. This is in accordance with the observations by Zenit et al. (1997).

Different pairs of particles were used, for a range of initial angles, and for various incidence angles. The properties of the spheres tested are shown in table 1 , which includes values of the particle diameter, $d$, the particle to fluid density ratio, $\rho_{p} / \rho_{f}$, the particle terminal velocity, $u_{t}$, and the particle terminal Reynolds number, $R e_{t}$. The fluid used for all of the experimental measurements was de-ionized water. 


\begin{tabular}{lcccr}
\hline Material & $d(\mathrm{~mm})$ & $\rho_{p} / \rho_{f}$ & $u_{t}\left(\mathrm{~cm} \mathrm{~s}^{-1}\right)$ & $R e_{t}$ \\
Glass $\times$ & 3.00 & 2.54 & 31.8 & 954 \\
Glass $\square$ & 4.10 & 2.54 & 36.8 & 1338 \\
Glass $\circ$ & 6.00 & 2.54 & 47.4 & 2583 \\
Steel $\diamond$ & 4.50 & 7.78 & 89.6 & 3665 \\
Nylon $\triangle$ & 6.35 & 1.14 & 13.6 & 785 \\
TABLE 1. Properties of particles used in experiments. \\
\hline
\end{tabular}

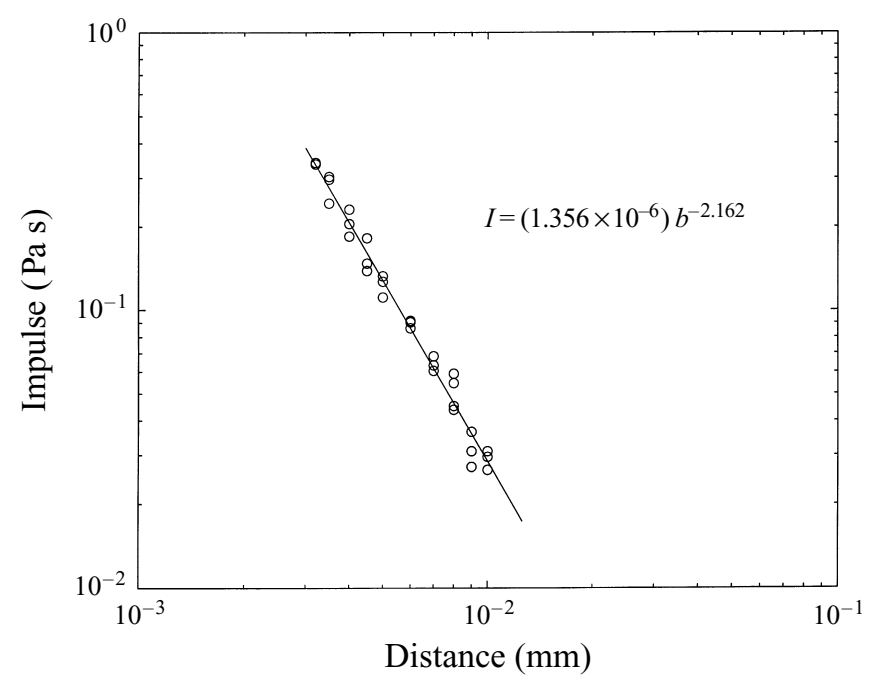

FIGURE 3. Measured impulse pressure as a function of distance from the wall, $b$. Acceleration of the particle produced by the collision of a particle of the same diameter and density; $6 \mathrm{~mm}$ glass particles immersed in water. Speed of the target particle after impact is $u_{\tau}=70.4 \mathrm{~mm} \mathrm{~s}^{-1}$.

The impulse pressure was calculated by

$$
I=\int_{0}^{\tau} P(t) \mathrm{d} t
$$

where $P(t)$ is the pressure signal, and $\tau$ is the duration of the pressure pulse, which is measured from the time of pressure increase until the first zero-cross of the signal after the main pulse. The secondary oscillations were neglected in the reduction of the data (Zenit 1997).

\section{Results}

Figure 3 shows the impulse pressure measured for a pair of $6 \mathrm{~mm}$ glass spheres as a function of the initial distance from the wall, $b$. The impact velocity for this experiment was constant by choosing an initial angle of release for the impact particle. The solid line shows the power law fit of the data. The measured impulse is approximately proportional to the inverse of the distance squared.

Figure 4 shows the measured impulse pressure as a function of the velocity of the target particle, $u_{\tau}$, for $6 \mathrm{~mm}$ glass particles. For this case the distance between the target particle and the wall was kept fixed at $b / d=0.63$. The velocity plotted on the horizontal axis is the velocity acquired by the target particle as a result of the 


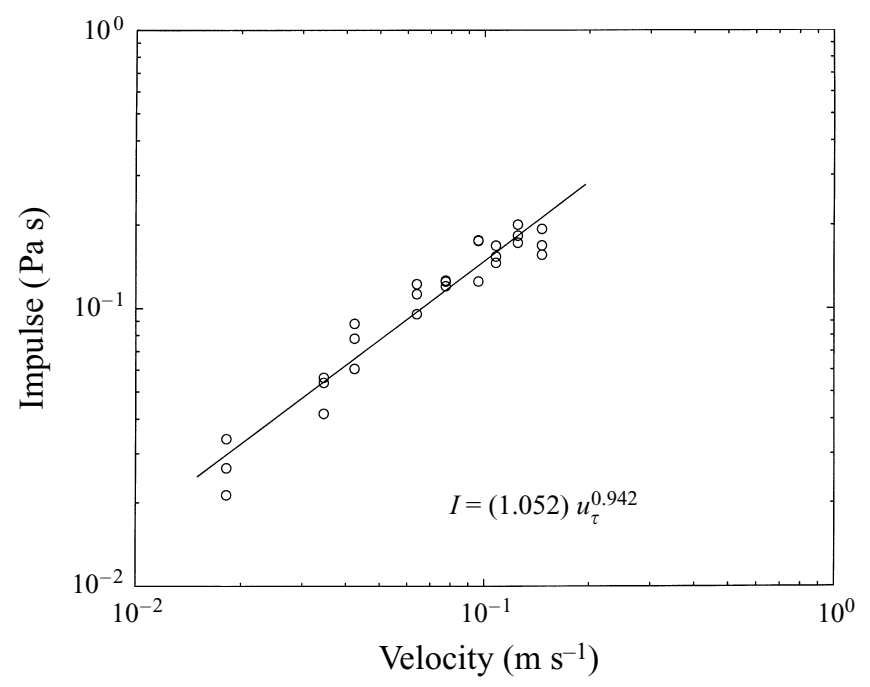

FIGURE 4. Measured impulse pressure as a function of impact velocity, $u_{\tau}$; distance from the wall, $b / d=0.63 ; 6 \mathrm{~mm}$ glass particles immersed in water.

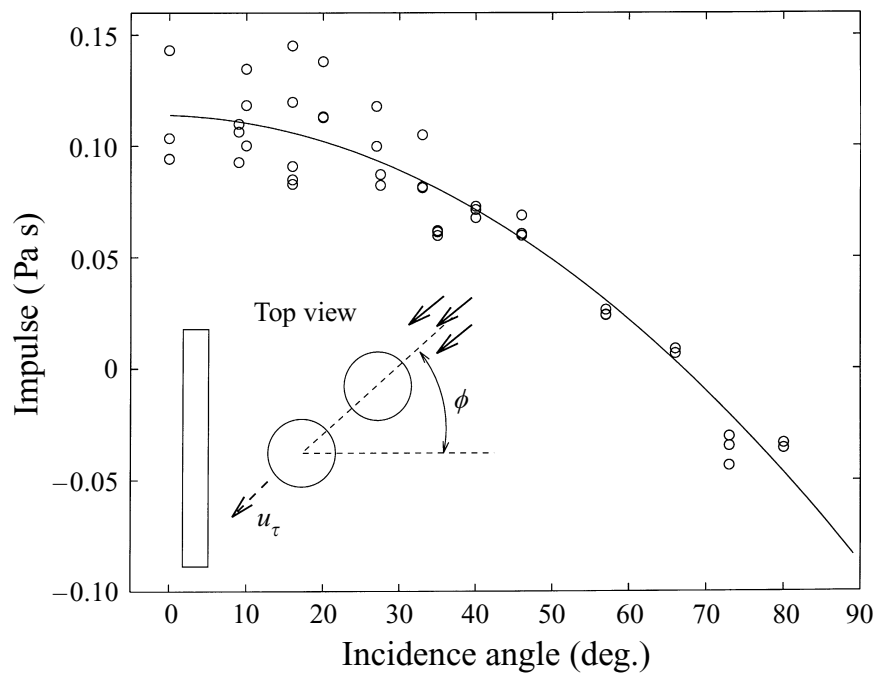

FIGURE 5. Measured impulse pressure as a function of incidence angle $\phi$ for $6 \mathrm{~mm}$ glass particles immersed in water; distance from the wall, $b / d=0.65$, and $u_{\tau}=95 \mathrm{~mm} \mathrm{~s}^{-1} ; 6 \mathrm{~mm}$ glass particles immersed in water. The solid line shows the best fit of the data.

collision. As expected, the magnitude of the impulse increases with the velocity. The impulse appears to increase linearly with $u_{\tau}$.

Experimental results were obtained also for different incident angles of the target particle with respect with the wall, for a fixed value of the distance $b / d$ and the velocity $u_{\tau}$. An angle $\phi=0$ represents an incidence motion normal to the wall, and $\phi=90^{\circ}$ is parallel to the wall. Figure 5 shows the measured impulse for $6 \mathrm{~mm}$ glass particles as a function of the angle $\phi$. For this particular experimental case $b / d=0.65$ and $u_{\tau}=95 \mathrm{~mm} / \mathrm{s}$.

Figure 6 presents the impulse as a function of the distance from the wall for three different diameters of glass spheres. The magnitude of the measured impulse is 


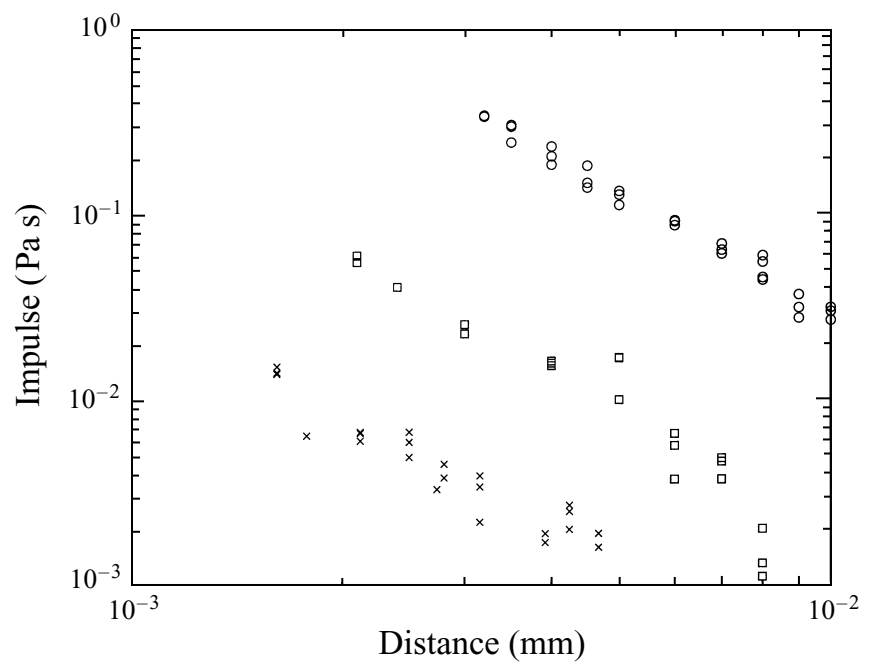

FiguRE 6. Comparison of the measured impulse pressure as a function of distance from the wall, $b$, for different diameter glass particles immersed in water: $6 \mathrm{~mm}$ glass $(\circ), u_{\tau}=70.4 \mathrm{~mm} \mathrm{~s}^{-1} ; 4.1 \mathrm{~mm}$ glass $(\square), u_{\tau}=59.6 \mathrm{~mm} \mathrm{~s}^{-1} ; 3 \mathrm{~mm}$ glass $(\times), u_{\tau}=27.9 \mathrm{~mm} \mathrm{~s}^{-1}$.

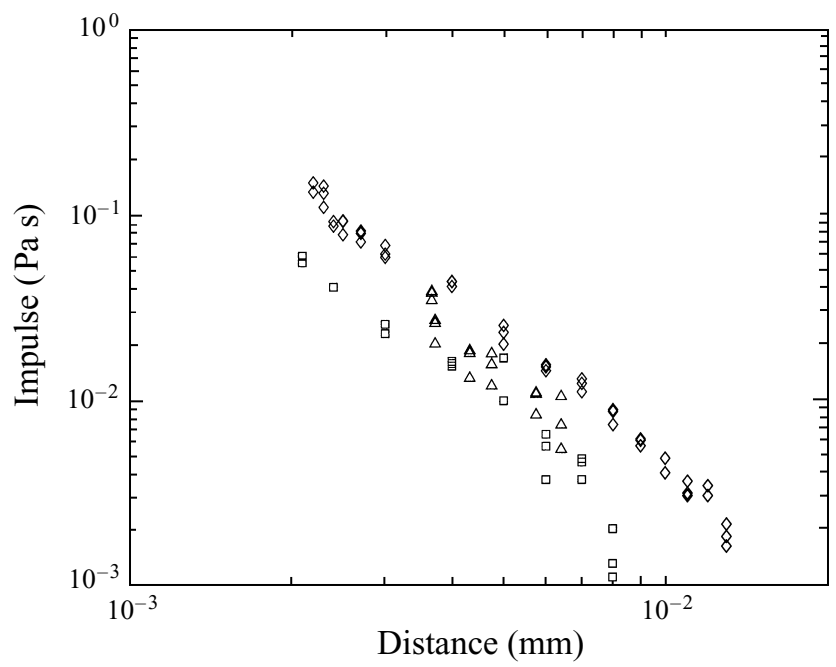

FIGURE 7. Comparison of the measured impulse pressure as a function of distance from the wall, $b$, for different density particles immersed in water: $4.1 \mathrm{~mm}$ glass $(\square), u_{\tau}=59.6 \mathrm{~mm} \mathrm{~s}^{-1} ; 4.5 \mathrm{~mm}$ steel $(\diamond), u_{\tau}=93.4 \mathrm{~mm} \mathrm{~s}^{-1} ; 6.35 \mathrm{~mm}$ nylon $(\triangle), u_{\tau}=20.0 \mathrm{~mm} \mathrm{~s}^{-1}$.

greatest for the largest particle; however, the velocities $u_{\tau}$ for these three cases differ. For the experimental setup, the control over $u_{\tau}$ is limited to a modest range for each type of particle; hence, it was not possible to match velocities $u_{\tau}$ for different particles. Figure 7 shows the measurements obtained for particles with different densities. Again, the target velocity, $u_{\tau}$, varies for each particle density.

However, if the measured impulses are normalized by $\hat{I}=I /\left(\rho_{f} u_{\tau} d\right)$, and the distance from the wall is normalized as $\hat{b}=b / d$, much of the data collapse into a single band, as shown in figure 8 . All particles show the same quadratic decay of the impulse with distance from the wall; however, for the same distance $b / d$ the measurements 


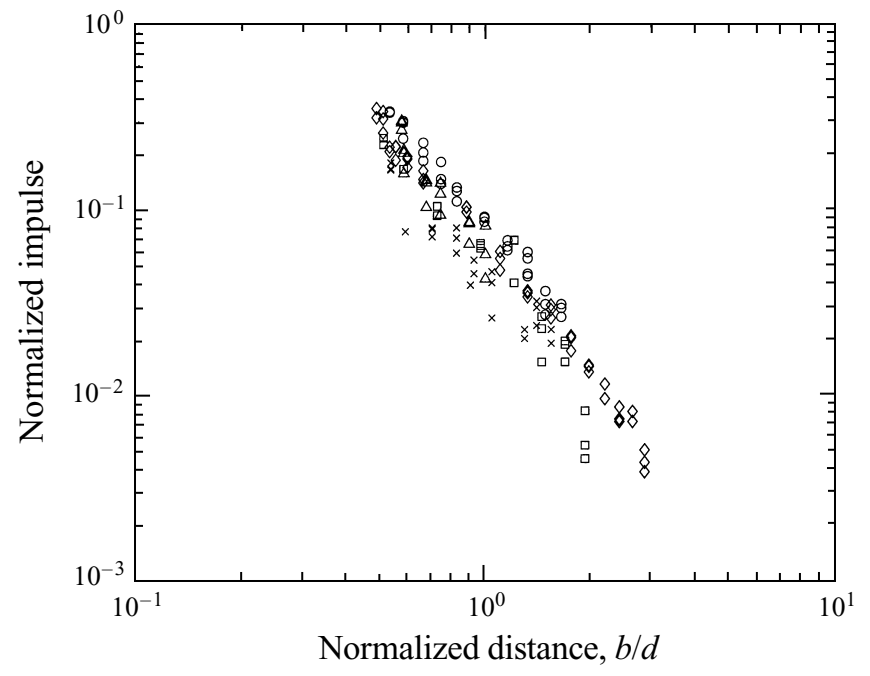

FIGURE 8. Comparison of the normalized impulse pressure, $\hat{I}$, as a function of normalized distance from the wall, $b / d$, for all tested particles. $6 \mathrm{~mm}$ glass $(\circ), R e_{\tau}=384 ; 4.1 \mathrm{~mm}$ glass $(\square), R e_{\tau}=222$; $3 \mathrm{~mm}$ glass $(\times), R e_{\tau}=76 ; 4.5 \mathrm{~mm}$ steel $(\diamond), R e_{\tau}=382 ; 6.25 \mathrm{~mm}$ nylon $(\triangle), R e_{\tau}=115$.

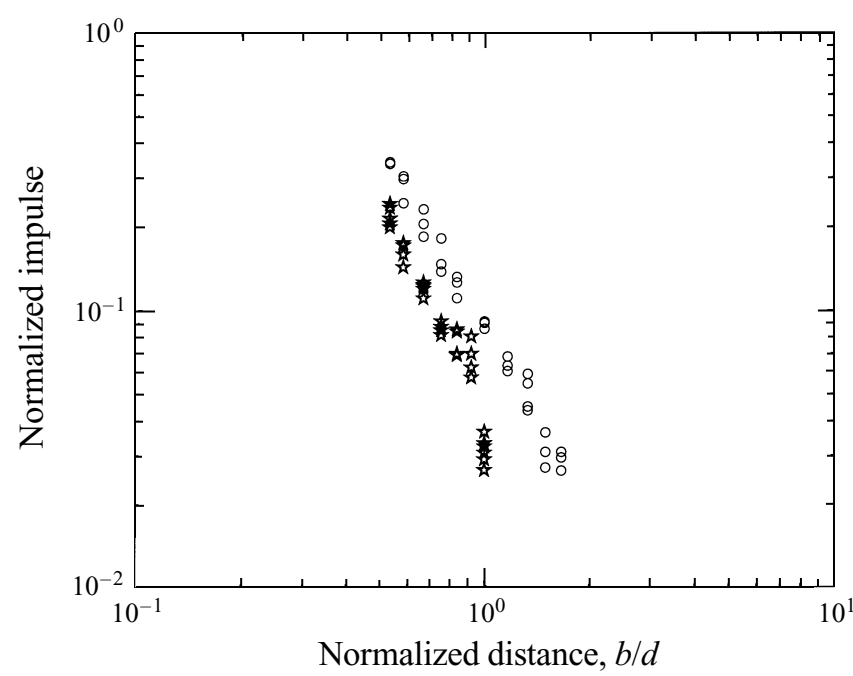

FIGURE 9. Normalized impulse pressure, $\hat{I}$, as a function of normalized distance from the wall, $b / d$, for a $6 \mathrm{~mm}$ glass particle using two different impact particles: $6.35 \mathrm{~mm}$ nylon $(\star), R e_{\tau}=109 ; 6 \mathrm{~mm}$ glass (०), $R e_{\tau}=384$.

occurring at higher values of impact Reynolds number, $\operatorname{Re}_{\tau}=\left(d \rho_{f} u_{\tau}\right) / \mu_{f}$, appear to have a higher normalized impulse.

\subsection{Measurements with dissimilar particles}

To obtain measurements of the pressure pulses generated by the same particles under different accelerations, experiments with pendulum pairs of dissimilar particles were performed. By changing the density of the impact particle, a new range of velocities $u_{\tau}$ can be achieved for the same target particle.

Figure 9 shows the impulse produced by a $6 \mathrm{~mm}$ glass particle being hit by 


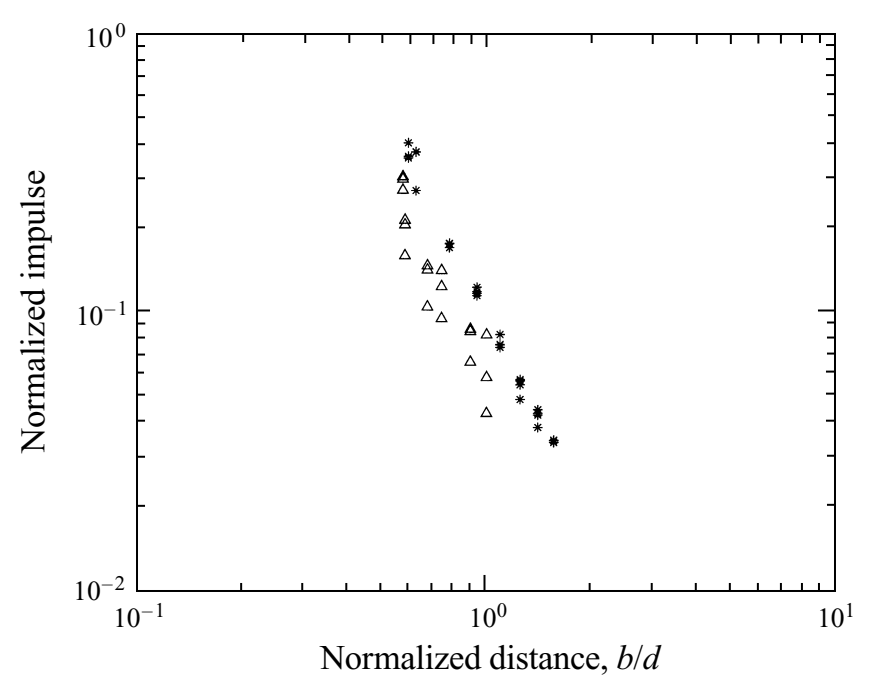

FIGURE 10. Normalized impulse pressure, $\hat{I}$, as a function of normalized distance from the wall, $b / d$, for a $6.35 \mathrm{~mm}$ using two different impact particles: $6 \mathrm{~mm}$ glass $(*), R e_{\tau}=577 ; 6.35 \mathrm{~mm}$ nylon $(\triangle)$, $R e_{\tau}=115$.

a $6.35 \mathrm{~mm}$ nylon particle. For comparison, data for two $6 \mathrm{~mm}$ glass spheres are also shown. Clearly, the rate of decay of the impulse for increasing distance is approximately the same for both cases. However, the magnitude for the lower velocity $u_{\tau}$ (lighter impact particle) is smaller for the same distance, $b / d$.

The same behaviour is observed in figure 10. Here the reversed case is shown: a $6.35 \mathrm{~mm}$ nylon particle is being accelerated by the collision of a $6 \mathrm{~mm}$ glass particle. The impulse produced by this configuration is greater than when the acceleration is produced by an identical (6.35 $\mathrm{mm}$ nylon) particle for the same distance, $b / d$. These experiments indicate the influence of the impact Reynolds number, $R e_{\tau}$, on the magnitude of the transmitted impulse. Experiments with other pairs of dissimilar particles were performed, indicating the same behaviour.

\section{Pressure-impulse theory}

To further investigate the nature of these pressure pulses, an analysis was carried out using the impulse pressure theory. The concept of impulse pressure, or impulsive pressure, is well known (Lamb 1945; Batchelor 1967), although it rarely appears in the multi-phase flow literature. A sudden acceleration of a solid boundary changes the pressure field in the surrounding fluid. If the change of velocity is the result of an impact, the pressure rises and falls in a short period of time generating large pressure gradients causing the velocity of the fluid to change.

Since the change of velocity during this impulsive event takes place during a short period of time, the nonlinear convective terms in the equation of motion are negligible compared with the time derivative. Thus, the equations of motion reduce to

$$
\frac{\partial \boldsymbol{u}}{\partial t}=-\frac{1}{\rho_{f}} \nabla P
$$

assuming an inviscid and incompressible flow. This equation can be time-integrated 


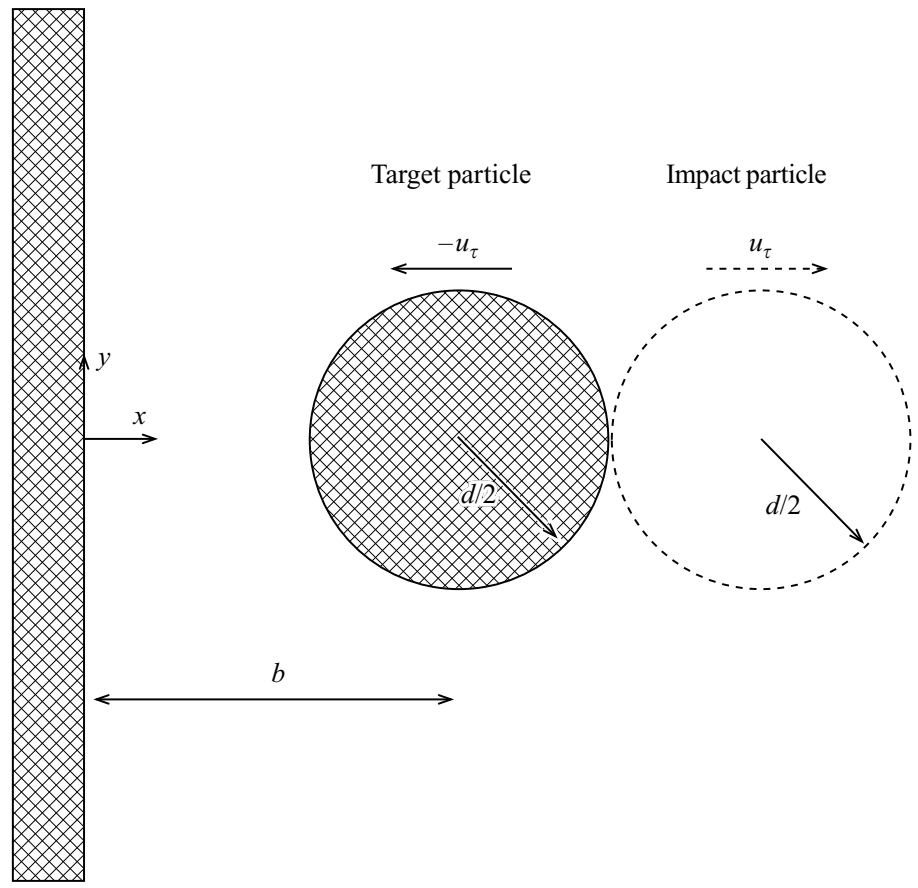

FIGURE 11. Schematic of the impulsive motion of a sphere near a wall in an inviscid irrotational flow.

through the impact time duration from $t=0$ to $t=\tau$ to obtain

$$
\boldsymbol{u}_{\tau}-\boldsymbol{u}_{0}=-\frac{1}{\rho_{f}} \nabla I
$$

where $\boldsymbol{u}_{0}$ and $\boldsymbol{u}_{\tau}$ are the particle velocities before and after the collision and $I$ is the pressure impulse (as defined in equation (2.1)). If the flow is incompressible, then it follows that

$$
\nabla^{2} I=0 .
$$

Thus the pressure generated by the impulsive motion of a body is governed by Laplace's equation, and resembles the velocity potential for the steady potential flow around that same body.

Consider the domain shown in figure 11. A spherical particle, of diameter $d$, is positioned at a certain distance, $b$, away from a solid wall. The fluid is incompressible and is at rest for $t \leqslant 0$. If at $t=0$ the particle is accelerated instantaneously from rest to a velocity $u_{\tau}$, the impulse pressure equation (4.3) can be solved using the following boundary conditions:

at the wall, $\boldsymbol{n} \cdot \nabla I=0$;

far away from the wall, $I=0(\boldsymbol{n} \cdot \nabla I=0$, could also be considered $)$;

on the surface of the particle, $\boldsymbol{n} \cdot \nabla I=\rho_{f} u_{\tau} \cos \theta$.

An approximate solution of the impulse produced by a sphere suddenly accelerated towards a wall can be obtained by superposing two doublets that are mirror images. The impulse, $I$, produced by a sphere, of diameter $d$, given a sudden change $-u_{\tau}$ in its velocity in the $x$-direction towards a wall, positioned initially at $x=+b, y=0$, is 


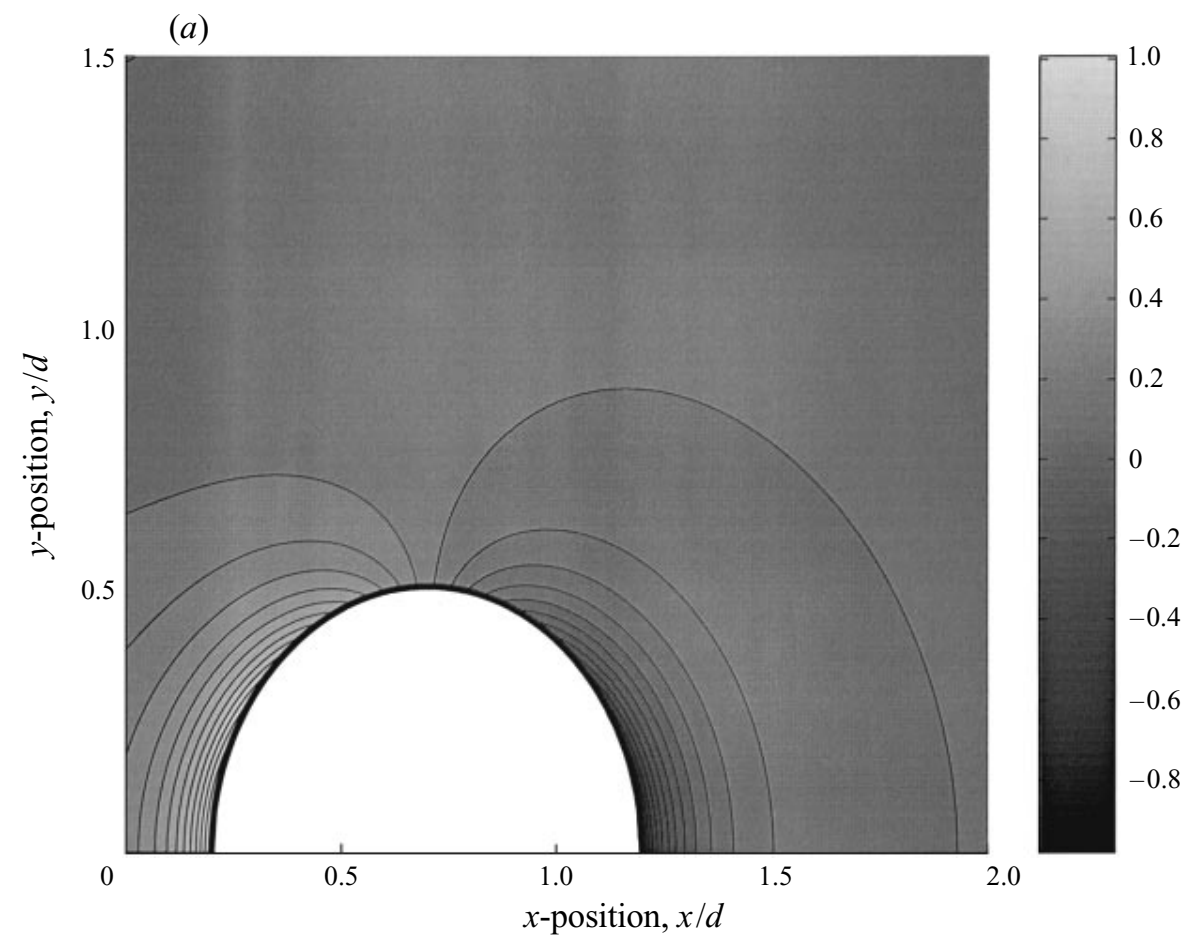

(b)

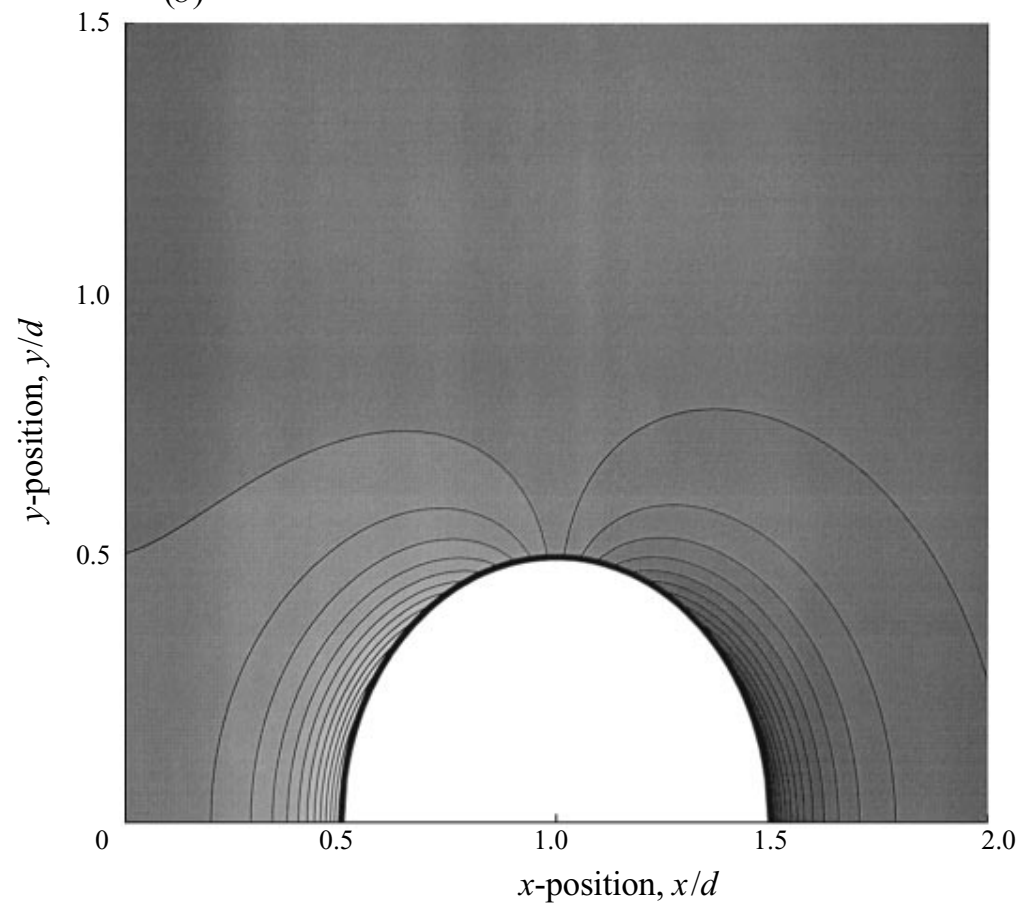

FIGURE $12(a, b)$. For caption see facing page. 
(c)

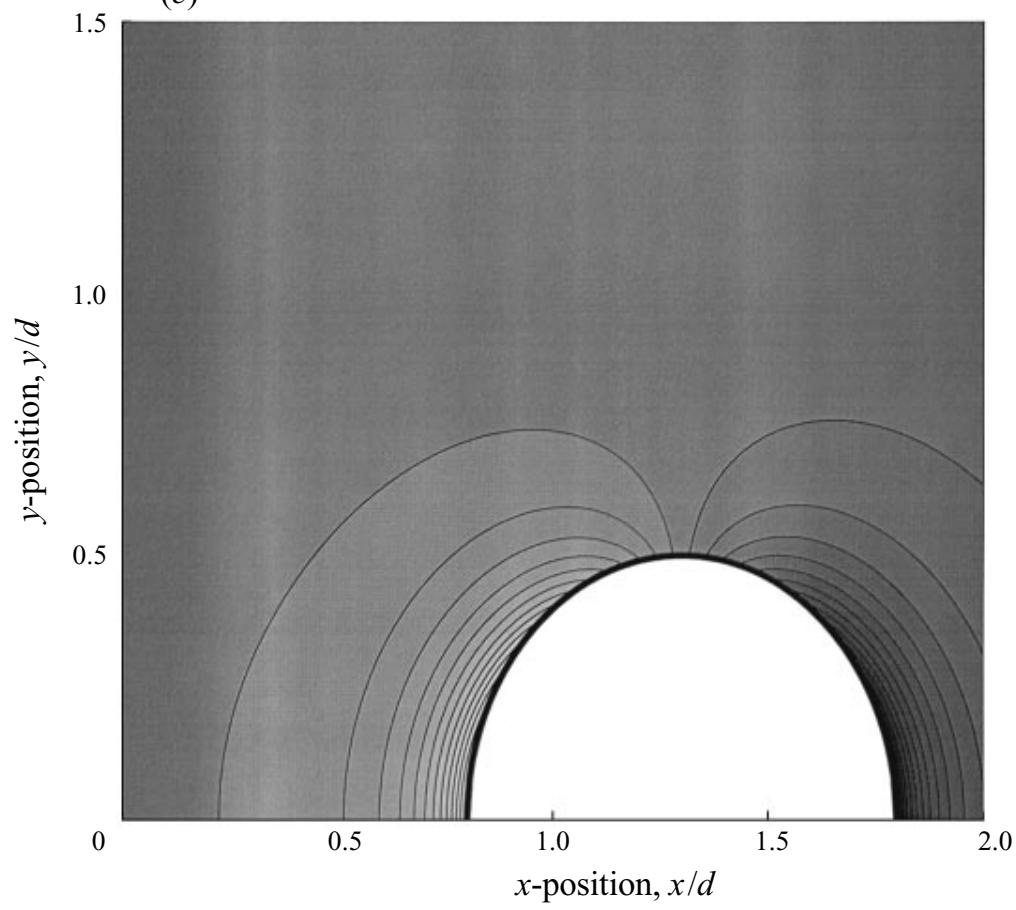

FIGURE 12. Impulse distribution for different $b / d$ ratios. The impulse is normalized by $\rho_{f} u_{\tau} d$. (a) $b / d=0.7,(b) b / d=1.0,(c) b / d=1.3$.

given by

$$
I=\frac{1}{2} \rho_{f} u_{\tau}\left(\frac{d}{2}\right)^{3}\left(\frac{x+b}{\left((x+b)^{2}+y^{2}\right)^{3 / 2}}-\frac{x-b}{\left((x-b)^{2}+y^{2}\right)^{3 / 2}}\right) .
$$

The impulse pressure distribution is shown in figure 12 for different $b / d$ ratios. The point $x / d=0$ represents the position of the wall. The solution using two doublets is not an exact solution for the flow of a particle approaching a wall because the method does not precisely satisfy the boundary condition on the surface of the sphere, especially when the sphere is close to the wall.

The exact solution of this problem can be obtained using a technique similar to one used by Landweber \& Shahshahan (1992), when two spheres approach each other in an inviscid flow. By adding an infinite series of image doublets, the boundary condition on the surface of the spheres can be satisfied exactly for any $b / d$. However, this procedure requires large summations when the two particles are very close to each other. The construction above should represent the essence of the mechanics of the radiated pulse caused by a suddenly accelerated particle near a boundary.

The magnitude of the impulse pressure depends also on the angle of the impulsive motion with respect to the wall. Using $\phi$ as the angle between the impulsive motion direction and the vector normal to the wall, equation (4.4) becomes

$$
I=\frac{1}{2} \rho_{f} u_{\tau}(d / 2)^{3}\left(\frac{(x+b) \cos \phi-y \sin \phi}{\left((x+b)^{2}+y^{2}\right)^{3 / 2}}-\frac{(x-b) \cos \phi+y \sin \phi}{\left((x-b)^{2}+y^{2}\right)^{3 / 2}}\right) .
$$




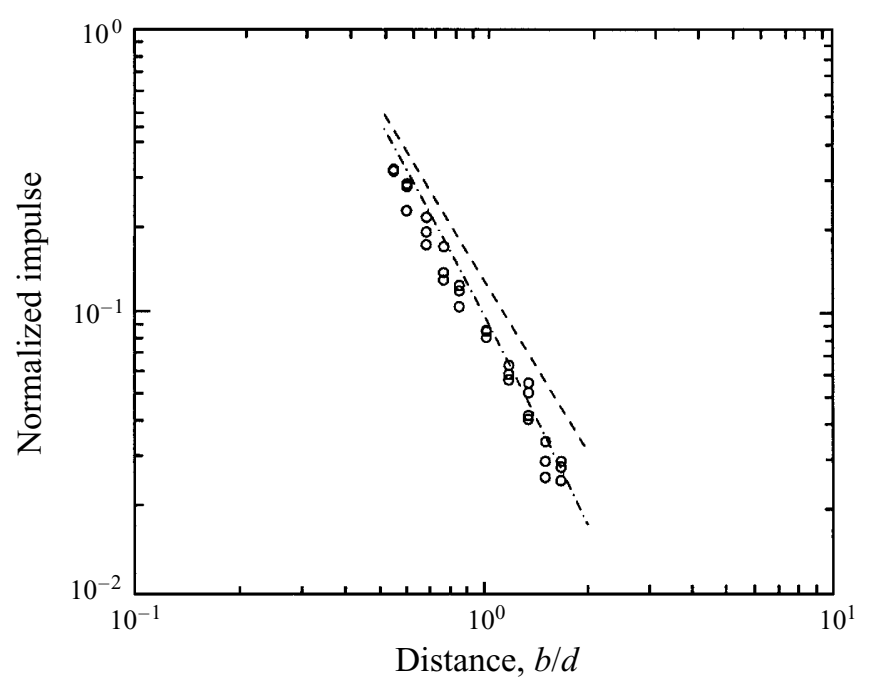

FiguRE 13. Comparison of the impulse pressure model prediction with experimental measurements. Normalized impulse as a function of distance from the wall for $6 \mathrm{~mm}$ glass (०), $R e_{\tau}=384$. Equation (5.1) $(---)$; equation (5.3) $(-\cdot-)$.

\section{Comparisons}

Comparisons can be made between the experimental results and the predictions from impulse-pressure theory. Equation (4.4) is re-written to express the impulse at the wall as a function of $b / d$. Normalizing by $\rho_{f} u_{\tau} d$ leads to

$$
\hat{I}_{w}=\frac{I(x=0, y=0)}{\rho_{f} u_{\tau} d}=\frac{1}{8}\left(\frac{d}{b}\right)^{2} .
$$

The dashed line in figure 13 shows the impulse calculated from this expression. For simplicity, only the results for the $6 \mathrm{~mm}$ glass spheres are shown in this figure. Clearly, the model appears to predict the decay of the impulse with distance, and over-predicts slightly the magnitude of the impulse.

In the experiment two particles collide. Therefore, a more appropriate model can be obtained, in an approximate solution, by the interaction of two pairs of doublets. As depicted in figure 11, the doublet modelling the second particle, the impact particle, is placed at $x=b+d$ with strength $\rho_{f} u_{\tau}$, with its respective mirror image placed at $x=-(b+d)$ with strength $-\rho_{f} u_{\tau}$. Therefore,

$$
\begin{aligned}
I=\frac{1}{2} \rho_{f} u_{\tau}(d / 2)^{3}\left(\frac{x+b}{\left((x+b)^{2}+y^{2}\right)^{3 / 2}}-\frac{x+b+d}{\left((x+b+d)^{2}+y^{2}\right)^{3 / 2}}\right. \\
\left.-\frac{x-b}{\left((x-b)^{2}+y^{2}\right)^{3 / 2}}+\frac{x-b-d}{\left((x-b-d)^{2}+y^{2}\right)^{3 / 2}}\right) .
\end{aligned}
$$

Thus, the normalized impulse at the wall reduces to

$$
\hat{I}_{w}=\frac{1}{8}\left(\frac{d}{b}\right)^{2}\left(\frac{2 b / d+1}{(b / d+1)^{2}}\right) .
$$

The dashed-dotted line in figure 13 shows the calculated impulse for the case of two doublets with their respective images. The prediction matches the experimental 


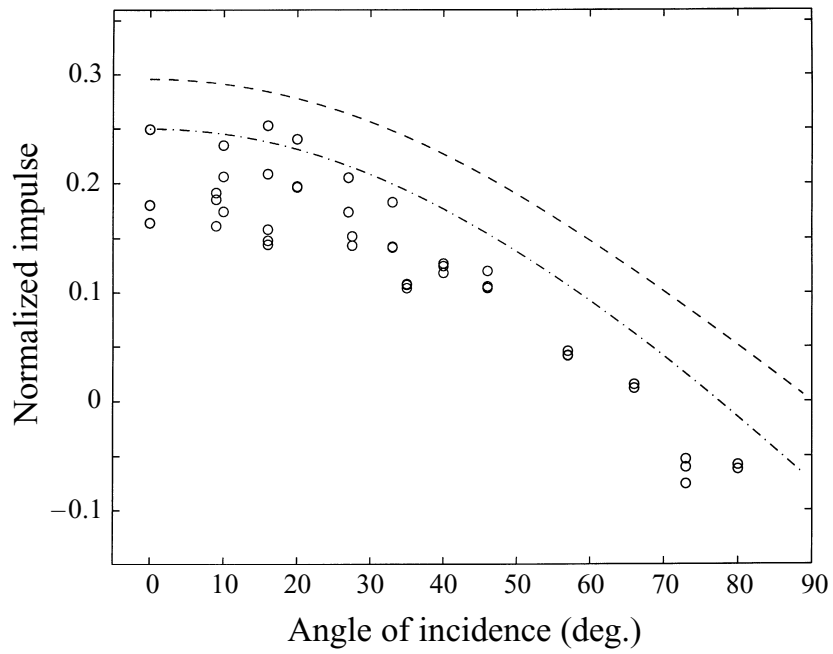

FIGURE 14. Comparison between theory and experiments of the normalized impulse pressure, $\hat{I}$, as a function of angle of incidence $\phi .6 \mathrm{~mm}$ glass (o), $R e_{\tau}=518$. Equation (5.4) $(---)$; equation (5.6) $(-\cdot-)$.

measurements better. Note that this expression neglects the sphere-sphere interaction of the collision pair.

Examining the dependence on the angle of incidence, equation (4.5) leads to a normalized impulse at the wall,

$$
\hat{I}_{w}=\frac{1}{8}\left(\frac{d}{b}\right)^{2} \cos \phi
$$

This result is shown in figure 14 by the dashed line. The experimental results obtained for the $6 \mathrm{~mm}$ glass beads with the same parameters are shown in the figure. The model appears to predict the decrease of the impulse for increasing angles of incidence but over-predicts the magnitude of the impulse.

The two-particle and four-doublet approach that led to equation (5.1), leads in the case of an oblique collision to

$$
\begin{array}{r}
I=\frac{1}{2} \rho_{f} u_{\tau}(d / 2)^{3}\left(\frac{(x+b) \cos \phi-y \sin \phi}{\left((x+b)^{2}+y^{2}\right)^{3 / 2}}-\frac{(x-b) \cos \phi+y \sin \phi}{\left((x-b)^{2}+y^{2}\right)^{3 / 2}}\right. \\
-\frac{(x+b+d \cos \phi) \cos \phi-(y-d \sin \phi) \sin \phi}{\left((x+b+d \cos \phi)^{2}+(y-d \sin \phi)^{2}\right)^{3 / 2}} \\
\left.+\frac{(x-b-d \cos \phi) \cos \phi+(y-d \sin \phi) \sin \phi}{\left((x-b-d \cos \phi)^{2}+(y-d \sin \phi)^{2}\right)^{3 / 2}}\right)
\end{array}
$$

and to an impulse at the wall given by

$$
\hat{I}_{w}=\frac{1}{8}\left(\frac{d}{b}\right)^{2} \cos \phi\left(1-\frac{(1+(d / b) \cos \phi)+(d / b) \sin \phi \tan \phi}{\left((1+(d / b) \cos \phi)^{2}+((d / b) \sin \phi)^{2}\right)^{3 / 2}}\right) .
$$

The dashed-dotted line in figure 14 shows the prediction from this expression. The model appears to predict the magnitude of the impulse better than equation (5.1) and does predict the negative impulse found in the experimental measurements for wide angles; however, it still over-estimates the magnitude for all angles. A refined model is described in the Appendix. 


\title{
6. Summary and conclusions
}

The radiated pulses reported by Zenit et al. (1997) were reproduced in a controlled fashion. Binary collisions were generated using a dual pendulum. One of the particles was suspended at rest at a particular distance from the transducer, while the other was swung from a specified initial angle. The collision produced an impulsive acceleration of the target particle, which transmitted a pressure front from the particle to the transducer through the interstitial fluid. Measurements of pressure pulses produced by binary collisions were obtained for a variety of particles and for a range of impact velocities and incident angles. The magnitude of the radiated impulse was found to be related to the particle velocity, the particle diameter and the distance from the wall. When the measured impulses are normalized by $\rho_{f} u_{\tau} d$ the data appear to collapse to a single curve. Particles with a small $R e_{\tau}$ (approximately less than 200) deviate from this trend, producing smaller values of the impulse for the same distance $b / d$. Hence, for smaller velocities, the viscous effect may be more important, thereby introducing other scaling parameters to the problem. Also, since the magnitude of the generated pressure pulses was small for the attainable velocities, measurements were only possible close to the transducer. Greater impulsive velocities for the light particles were achieved by using a denser impact particle. These measurements confirmed that for the same target particle, a decrease of the impact velocity decreased the measured impulse.

To predict the measured impulse, an analysis was performed using pressure-impulse theory. By neglecting both convective and viscous effects and integrating through the impact time, the incompressible equation of motion reduced to Laplace's equation for the pressure impulse. Approximate expressions for the solution of the impulse field of a solid sphere near a wall were obtained using the superposition of doublets. The prediction from the pressure-impulse theory agrees with the experimental measurements using either a doublet and its image, or using a pair of doublets and their images, even though the solid boundary condition on the spheres is only satisfied approximately.

The authors are grateful to Professors Charles Campbell and Chris Brennen for helpful discussions and suggestions. The National Council for Science and Technology of Mexico (CONACYT) is acknowledged for supporting R. Zenit during his graduate sojourn at the California Institute of Technology. The authors wish to thank Professor $\mathrm{R}$. Mei for his careful review, which directed us to a sign error in an earlier version of this manuscript.

\section{Appendix. Refined model with sphere-sphere interactions and leading-order wall effects}

\author{
by Ludwig C. Nitsche \\ Chemical Engineering, University of Illinois at Chicago, 810 S. Clinton, Chicago, \\ IL 60607, USA
}

Equations (5.2), (5.3), (5.5) and (5.6) embody two levels of approximation.

(i) Near field: neglect of hydrodynamic interactions between the touching spheres (whether real or images).

(ii) Far field: neglect of wall effects by which the image spheres introduce errors in the boundary conditions for the original collision pair, and vice versa.

In order to assess the comparison between theory and experiment, it seems useful to do a refined calculation that at least partially avoids these approximations. 
Both effects would be amenable to the method of reflections for arbitrary angle of incidence $\phi$. We shall consider the axisymmetric case of figure $13(\phi=0)$ because the near-field correction - which seems to require going to higher order - can then be treated (more efficiently) by the method of images (Lamb 1945). We shall be satisfied with just the (leading term of the) first reflection for the far-field correction.

Attacking first the sphere-sphere interaction by itself, consider one sphere moving with unit velocity while its (touching) collision partner remains fixed. (This is simply a decomposition of the mathematical problem based upon linearity, and should not be interpreted as applying literally to the separation of the spheres after collision.) Making distances dimensionless with respect to the sphere radius, we define the positions of successive image dipoles (of strengths $\mu_{j}$, located along the axis of symmetry) in terms of their distances $f_{j}$ from the centre of the moving sphere. The distances and strengths are given by Lamb (1945, Art. 98): by this scheme each successive dipole cancels out the velocity disturbance produced on the surface of its surrounding sphere by the preceding dipole located in the other sphere. Starting with $f_{0}=0$ and $\mu_{0}=1$, successive steps yield

$$
\left.\begin{array}{rlrl}
f_{j-1} & =2-\frac{1}{2-f_{j-2}}, & \frac{\mu_{j-1}}{\mu_{j-2}} & =\frac{-1}{\left(2-f_{j-2}\right)^{3}}, \\
f_{j} & =\frac{1}{f_{j-1}}, & \frac{\mu_{j}}{\mu_{j-1}} & =\frac{-1}{f_{j-1}^{3}}
\end{array}\right\}(j=2,4,6, \ldots) .
$$

By adding to the flow field generated by the colliding spheres that due to a mirrorimage collision pair behind the wall, reflective symmetry eliminates the normal velocity component at the wall (boundary condition of no fluid penetration, slip being allowed for potential flow). Now each collision pair does, however, alter the velocity field felt over the surfaces of its mirror image - an error which, when corrected, represents the first-reflection correction for wall effects. Upon Taylor expanding the incident velocity disturbance about the centre of each sphere, we find at leading order just a uniform flow over the surface, which can be cancelled out simply by (slightly) altering the velocity of the sphere. This correction appears at order $(b / d)^{3}$. Additional correction terms would cancel out the linear, quadratic and higher-order variations of the incident disturbance field in the first reflection, but these would start at order $(b / d)^{4}$ and are here neglected (along with the higher reflections).

Because the target sphere and impact sphere experience (slightly) different incident disturbance fields, they are dealt with separately by breaking apart the collision problem into two subproblems; in each of these one sphere moves while the other remains fixed (figure 15). The new velocities ( $U$ for the target sphere vs. $V$ for the impact sphere) are chosen so as to cancel out the incident disturbance velocity vectors evaluated at the respective sphere centres - keeping in mind that the disturbance field itself emanates from an infinite sequence of dipoles within the mirror-image collision pair. Note that the unaltered velocities of the mirror-image collision pair suffice for computing the disturbance at leading order:

$$
\left.\begin{array}{l}
U=-1+\sum_{j=0}^{\infty} \mu_{j}\left\{\left[4(b / d)-f_{j}+2\right]^{-3}-\left[4(b / d)+f_{j}\right]^{-3}\right\}, \\
V=1+\sum_{j=0}^{\infty} \mu_{j}\left\{\left[4(b / d)-f_{j}+4\right]^{-3}-\left[4(b / d)+f_{j}+2\right]^{-3}\right\} .
\end{array}\right\}
$$




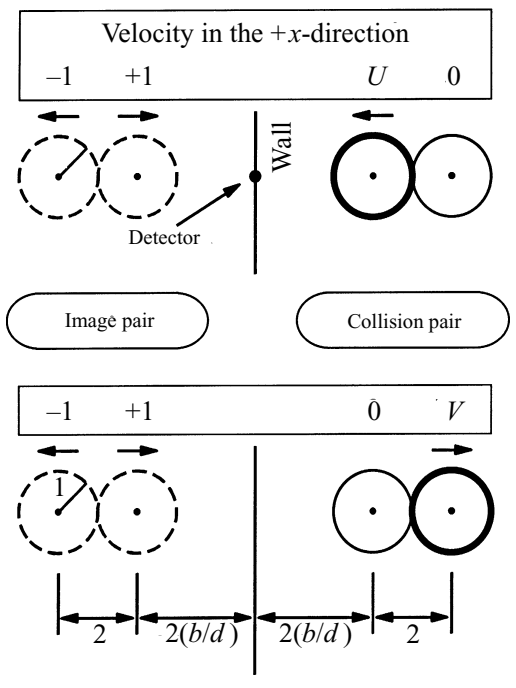

FIGURE 15. Decomposition into two subproblems of the sphere-sphere separation (immediately after collision) in the presence of the incident field emanating from the image pair of spheres. In each subproblem one sphere moves (with velocity $U$ or $V$ ) while its collision partner remains fixed.

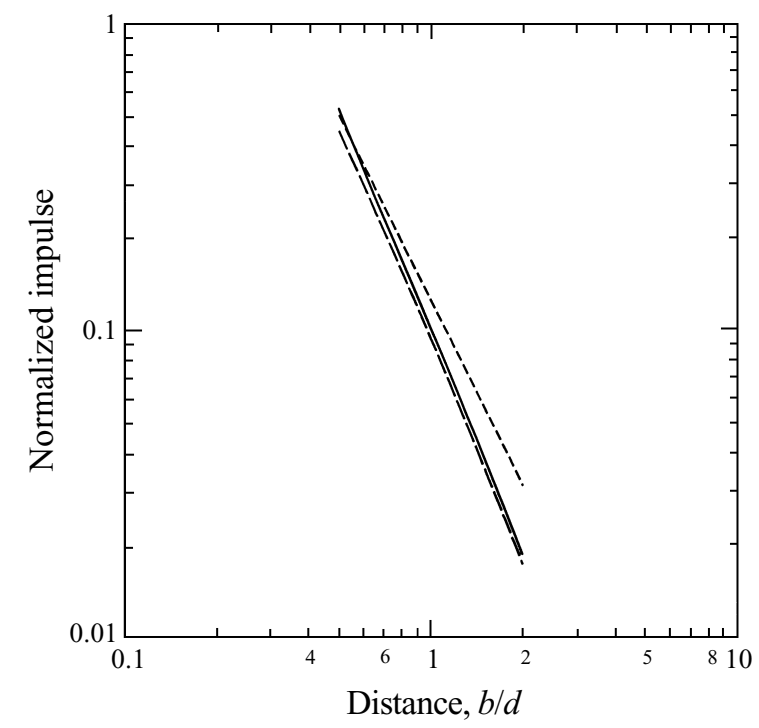

FIGURE 16. Comparison of the three theoretical models for the impulse pressure measured at the detector in the wall: equations (5.1) (- - ) and (5.3) (- - ) vs. (A 4) (-).

Here the impulse pressure field $I$ plays the role of the velocity potential. Evaluated at the detector one finds for the normalized impulse at the wall

$$
\begin{aligned}
\hat{I}_{w} & =\left\{\sum_{j=0}^{\infty} \frac{\mu_{j} / 2}{\left[2(b / d)+f_{j}\right]^{2}}\right\}-\left\{\sum_{j=0}^{\infty} \frac{\mu_{j} / 2}{\left[2(b / d)-f_{j}+2\right]^{2}}\right\}+O\left[(b / d)^{5}\right], \\
& =-U\left\{\sum_{j=0}^{\infty} \frac{\mu_{j} / 2}{\left[2(b / d)+f_{j}\right]^{2}}\right\}-V\left\{\sum_{j=0}^{\infty} \frac{\mu_{j} / 2}{\left[2(b / d)-f_{j}+2\right]^{2}}\right\}+O\left[(b / d)^{7}\right],
\end{aligned}
$$


with $U$ and $V$ given by equation (A 2). Both formulas treat the sphere-sphere interaction exactly; only the latter incorporates the leading wall-effect correction.

Figure 16 compares equation (A 4) with the curves for equations (5.1) and (5.3). The summations have been taken to 100th order, which is sufficient for accuracy within \pm 3 in the 7 th digit. Relative to the magnitude of $\hat{I}_{w}$ as given by equation (5.3), the correction for the sphere-sphere interaction (equation (A 3)) amounts to $5.2 \%$ when the target sphere touches the wall $(b / d=0.5)$, and levels off at $8.2 \%$ as $b / d \rightarrow \infty$, this being for touching spheres! The additional wall-effect contribution from equation (A 4 ) starts at $13.0 \%$ when $b / d=0.5$ and has already decayed to just $1.6 \%$ at a sphere-wall gap of one radius $(b / d=1)$. Thus - in the axisymmetric case $\phi=0$ - these results confirm the premise of Zenit \& Hunt that just the two doublets plus mirror images (equation (5.3)) should give a good representation of the data. Note that the more accurate curve is slightly farther from most of the data points, especially at small $(b / d)$.

\section{REFERENCES}

Batchelor, G. K. 1967 An Introduction to Fluid Dynamics. Cambridge University Press.

BAtchelor, G. K. 1988 A new theory of the instability of a uniform fluidized bed. J. Fluid Mech. 193, 75-110.

Cooker, M. J. \& Peregrine, D. H. 1995 Pressure-impulse theory for liquid impact problems. $J$. Fluid Mech. 297, 193-214.

LAMB, H. 1945 Hydrodynamics. Dover Publications.

LANDweber, L. \& Shahshahan, A. 1992 Added masses and forces on two bodies approaching central impact in an inviscid fluid. J. Ship Res. 36, 99-122.

LovalEnti, P. M. \& Brady, J. F. 1995 The temporal behaviour of the hydrodynamic force on a body in response to an abrupt change in velocity at small but finite Reynolds number. $J$. Fluid Mech. 293, 35-46.

Mei, R. \& Lawrence, C. J. 1996 The flow-field due to a body in impulsive motion. J. Fluid Mech. 325, 79-111.

ZENIT, R. 1997 Collisional mechanics in liquid-solid flows. PhD thesis, California Institute of Technology, Pasadena, California.

ZENit, R. \& HunT, M. L. 1997 Pressure front generated by the impulsive motion of a liquid resulting from a particle collision. In AICHE Annual Meeting Proceedings, Los Angeles, California.

Zenit, R. \& Hunt, M. L. 1998 Mechanics of immersed collisions of particles. Submitted to Trans. ASME: J. Fluids Engng

Zenit, R., Hunt, M. L. \& Brennen, C. E. 1997 Collisional particle pressure measurements in solid-liquid flows. J. Fluid Mech. 353, 261-283. 